\title{
Erratum: Collision-energy dependence of second-order off-diagonal and diagonal cumulants of net-charge, net-proton, and net-kaon multiplicity distributions in Au + Au collisions [Phys. Rev. C 100, $014902(2019)]$
}

J. Adam, L. Adamczyk, J. R. Adams, J. K. Adkins, G. Agakishiev, M. M. Aggarwal, Z. Ahammed, I. Alekseev, D. M. Anderson, R. Aoyama, A. Aparin, D. Arkhipkin, E. C. Aschenauer, M. U. Ashraf, F. Atetalla, A. Attri, G. S. Averichev, V. Bairathi, K. Barish, A. J. Bassill, A. Behera, R. Bellwied, A. Bhasin, A. K. Bhati, J. Bielcik, J. Bielcikova, L. C. Bland, I. G. Bordyuzhin, J. D. Brandenburg, A. V. Brandin, J. Bryslawskyj, I. Bunzarov, J. Butterworth, H. Caines, M. Calderón de la Barca Sánchez, D. Cebra, I. Chakaberia, P. Chaloupka, B. K. Chan, F-H. Chang, Z. Chang, N. Chankova-Bunzarova, A. Chatterjee $\odot$, S. Chattopadhyay, J. H. Chen, X. Chen, J. Cheng, M. Cherney, W. Christie, H. J. Crawford, M. Csanád, S. Das, T. G. Dedovich, I. M. Deppner, A. A. Derevschikov, L. Didenko, C. Dilks, X. Dong, J. L. Drachenberg, J. C. Dunlop, T. Edmonds, N. Elsey, J. Engelage, G. Eppley, R. Esha, S. Esumi, O. Evdokimov, J. Ewigleben, O. Eyser, R. Fatemi, S. Fazio, P. Federic, J. Fedorisin, Y. Feng, P. Filip, E. Finch, Y. Fisyak, L. Fulek, C. A. Gagliardi, T. Galatyuk, F. Geurts, A. Gibson, D. Grosnick, A. Gupta, W. Guryn, A. I. Hamad, A. Hamed, J. W. Harris, L. He, S. Heppelmann, S. Heppelmann, N. Herrmann, L. Holub, Y. Hong, S. Horvat, B. Huang, H. Z. Huang, S. L. Huang, T. Huang, X. Huang, T. J. Humanic, P. Huo, G. Igo, W. W. Jacobs, A. Jentsch, J. Jia, K. Jiang, S. Jowzaee, X. Ju, E. G. Judd, S. Kabana, S. Kagamaster, D. Kalinkin, K. Kang, D. Kapukchyan, K. Kauder, H. W. Ke, D. Keane, A. Kechechyan, M. Kelsey, Y. V. Khyzhniak, D. P. Kikoła, C. Kim, T. A. Kinghorn, I. Kisel, A. Kisiel, M. Kocan, L. Kochenda, L. K. Kosarzewski, L. Kramarik, P. Kravtsov, K. Krueger, N. Kulathunga Mudiyanselage, L. Kumar, R. Kunnawalkam Elayavalli, J. H. Kwasizur, R. Lacey, J. M. Landgraf, J. Lauret, A. Lebedev, R. Lednicky, J. H. Lee, C. Li, W. Li, W. Li, X. Li, Y. Li, Y. Liang, R. Licenik, T. Lin, A. Lipiec, M. A. Lisa, F. Liu, H. Liu, P. Liu, P. Liu, T. Liu, X. Liu, Y. Liu, Z. Liu, T. Ljubicic, W. J. Llope, M. Lomnitz, R. S. Longacre, S. Luo, X. Luo, G. L. Ma, L. Ma, R. Ma, Y. G. Ma, N. Magdy, R. Majka, D. Mallick, S. Margetis, C. Markert,

H. S. Matis, O. Matonoha, J. A. Mazer, K. Meehan, J. C. Mei, N. G. Minaev, S. Mioduszewski, D. Mishra, B. Mohanty, M. M. Mondal, I. Mooney, Z. Moravcova, D. A. Morozov, Md. Nasim, K. Nayak, T. K. Nayak, J. M. Nelson, D. B. Nemes, M. Nie, G. Nigmatkulov, T. Niida, L. V. Nogach, T. Nonaka, G. Odyniec, A. Ogawa, K. Oh, S. Oh, V. A. Okorokov, B. S. Page, R. Pak, Y. Panebratsev, B. Pawlik, D. Pawlowska, H. Pei, C. Perkins, R. L. Pintér, J. Pluta, J. Porter, M. Posik, N. K. Pruthi,

M. Przybycien, J. Putschke, A. Quintero, S. K. Radhakrishnan, S. Ramachandran, R. L. Ray, R. Reed, H. G. Ritter,

J. B. Roberts, O. V. Rogachevskiy, J. L. Romero, L. Ruan, J. Rusnak, O. Rusnakova, N. R. Sahoo, P. K. Sahu, S. Salur, J. Sandweiss, J. Schambach, W. B. Schmidke, N. Schmitz, B. R. Schweid, F. Seck, J. Seger, M. Sergeeva, R. Seto, P. Seyboth, N. Shah, E. Shahaliev, P. V. Shanmuganathan, M. Shao, F. Shen, W. Q. Shen, S. S. Shi, Q. Y. Shou, E. P. Sichtermann, S. Siejka, R. Sikora, M. Simko, J. Singh, S. Singha, D. Smirnov, N. Smirnov, W. Solyst, P. Sorensen, H. M. Spinka, B. Srivastava,

T. D. S. Stanislaus, M. Stefaniak, D. J. Stewart, M. Strikhanov, B. Stringfellow, A. A. P. Suaide, T. Sugiura, M. Sumbera,

B. Summa, X. M. Sun, Y. Sun, Y. Sun, B. Surrow, D. N. Svirida, P. Szymanski, A. H. Tang, Z. Tang, A. Taranenko,

T. Tarnowsky, J. H. Thomas, A. R. Timmins, D. Tlusty, T. Todoroki, M. Tokarev, C. A. Tomkiel, S. Trentalange, R. E. Tribble, P. Tribedy, S. K. Tripathy, O. D. Tsai, B. Tu, T. Ullrich, D. G. Underwood, I. Upsal, G. Van Buren, J. Vanek, A. N. Vasiliev, I. Vassiliev, F. Videbæk, S. Vokal, S. A. Voloshin, F. Wang, G. Wang, P. Wang, Y. Wang, Y. Wang, J. C. Webb, L. Wen, G. D. Westfall, H. Wieman, S. W. Wissink, R. Witt, Y. Wu, Z. G. Xiao, G. Xie, W. Xie, H. Xu, N. Xu, Q. H. Xu, Y. F. Xu, Z. Xu, C. Yang, Q. Yang, S. Yang, Y. Yang, Z. Ye, Z. Ye, L. Yi, K. Yip, I.-K. Yoo, H. Zbroszczyk, W. Zha, D. Zhang, L. Zhang, S. Zhang, S. Zhang, X. P. Zhang, Y. Zhang, Z. Zhang, J. Zhao, C. Zhong, C. Zhou, X. Zhu, Z. Zhu, M. Zurek, and M. Zyzak (STAR Collaboration)

(Received 22 October 2021; published 4 February 2022)

DOI: 10.1103/PhysRevC.105.029901

In the original paper, the first measurements of off-diagonal cumulants of net-charge, net-proton (a proxy for the netbaryon), and net-kaon (a proxy for the net-strangeness) were reported using the first phase of beam energy scan (BES-I) data from the Relativistic Heavy Ion Collider (RHIC). The second-order mixed-cumulant ratios between net-proton and net-kaon $\left(C_{p, k}=\sigma_{p, k}^{1,1} / \sigma_{k}^{2}\right)$ at different collision energies $\left(\sqrt{s_{N N}}=7.7-200 \mathrm{GeV}\right)$ show a good agreement with various model predictions. However, the mixed cumulants between net-charge and net-proton $\left(C_{Q, p}=\sigma_{Q, p}^{1,1} / \sigma_{p}^{2}\right)$, as well as the mixed cumulants between net-charge and net-kaon $\left(C_{Q, k}=\sigma_{Q, k}^{1,1} / \sigma_{k}^{2}\right)$, showed significant deviations from the model predictions. An increasing trend of these ratios as a function of collision energy in 0-5\% central events was reported. Triggered by the theory papers [1], we 


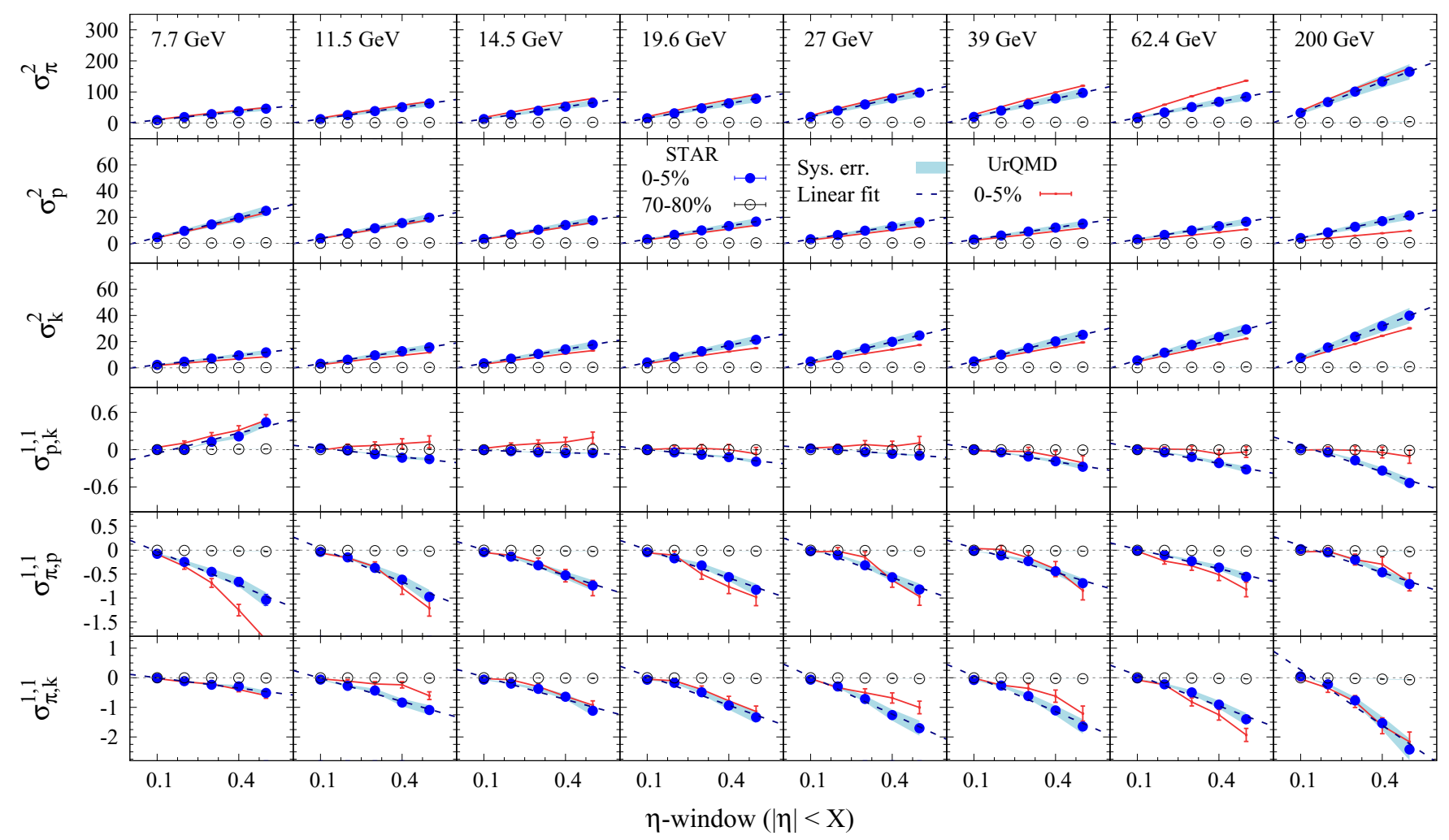

FIG. 2. The dependence of efficiency-corrected second-order diagonal and off-diagonal cumulants on the width of the $\eta$-window. The filled and open circles represent $0-5 \%$ and $70-80 \%$ central collisions respectively. The shaded band represents the systematic uncertainty. The statistical uncertainties are within the marker size and solid lines are UrQMD calculations.

realized that the excess correlations in $\sigma_{Q, p}^{1,1}$ and $\sigma_{Q, k}^{1,1}$ arise due to an artifact of assuming $Q$ and $p$ (or $k$ ) to be mutually exclusive variables while correcting for the particle reconstruction efficiency effects. In this erratum, we address this issue. We now present the new observables $\sigma_{\pi, k}^{1,1}$, and $\sigma_{\pi, p}^{1,1}$ that avoid the above assumption. The previously observed increasing trend with energy in $\sigma_{Q, k}^{1,1}$, and $\sigma_{Q, p}^{1,1}$ is no longer seen in the new observables of $\sigma_{\pi, k}^{1,1}$, and $\sigma_{\pi, p}^{1,1}$.

In the original paper, the efficiency correction for $\sigma_{p, k}^{1,1}, \sigma_{Q, p}^{1,1}$, and $\sigma_{Q, k}^{1,1}$ was performed using the binomial efficiency correction method $[2,3]$ assuming net-charge, net-proton, and net-kaon are mutually exclusive variables. In that case, the expression for the efficiency correction formula for $\sigma_{Q, p}^{1,1}$ is

$$
\sigma_{Q, p}^{1,1}(\text { Corrected })=\frac{1}{\epsilon_{Q} \epsilon_{p}}\left\langle n_{Q} n_{p}\right\rangle-\frac{1}{\epsilon_{Q}}\left\langle n_{Q}\right\rangle \frac{1}{\epsilon_{p}}\left\langle n_{p}\right\rangle
$$

Here $\langle\cdots\rangle$ represents average over events in a given centrality class. The $n_{Q}$ and $n_{p}$ are the measured net-charge and net-proton numbers within the acceptance of our measurement. The $\epsilon_{Q}$ and $\epsilon_{p}$ are the average efficiencies for inclusive charged particles and protons, respectively. A similar expression is also used for $\sigma_{Q, k}^{1,1}$. For inclusive charged particles no identification is performedonly the charge state is measured using the STAR time projection chamber (TPC) by measuring its helix. But for estimation of efficiency $\epsilon_{Q}$, the weighted average of tracking efficiencies of protons, pions, and kaons are used. Recently, we discovered that Eq. (1) is not valid for mutually inclusive variables such as those in $Q-p$ and $Q-k$ correlations. This is because inclusive charge particle multiplicity $\left(n_{Q}\right)$ contains both protons $\left(n_{p}\right)$ and kaons $\left(n_{k}\right)$. This introduces a self-correlation in the previously considered efficiency correction procedure. A detailed discussion of this issue can be found in Refs. [1,4].

To avoid this problem, we report the correlation between net-pion and net-proton $\left(\sigma_{\pi, p}^{1,1}\right)$ and between net-pion and net-kaon $\left(\sigma_{\pi, k}^{1,1}\right)$. This can help us address the problem of self-correlation in $C_{Q, p}$ and $C_{Q, k}$ [4]. The combination between net-proton and net-kaon was already published in the original paper. Pions have been selected within $0.4<p_{T}<1.6 \mathrm{GeV} / c$ using both TPC and time of flight (TOF). To select pions a cut $\left|n \sigma_{\pi}\right|<2$ and $0.01<m^{2}<0.06(\mathrm{GeV} / c)^{2}$ has been applied. Proton and kaon identifications are same as in the original paper. Using $\sigma_{\pi, p}^{1,1}, \sigma_{\pi, k}^{1,1}$, and $\sigma_{p, k}^{1,1}$ we can redefine the cumulant ratios $C_{Q, p}$ and $C_{Q, k}$ as 


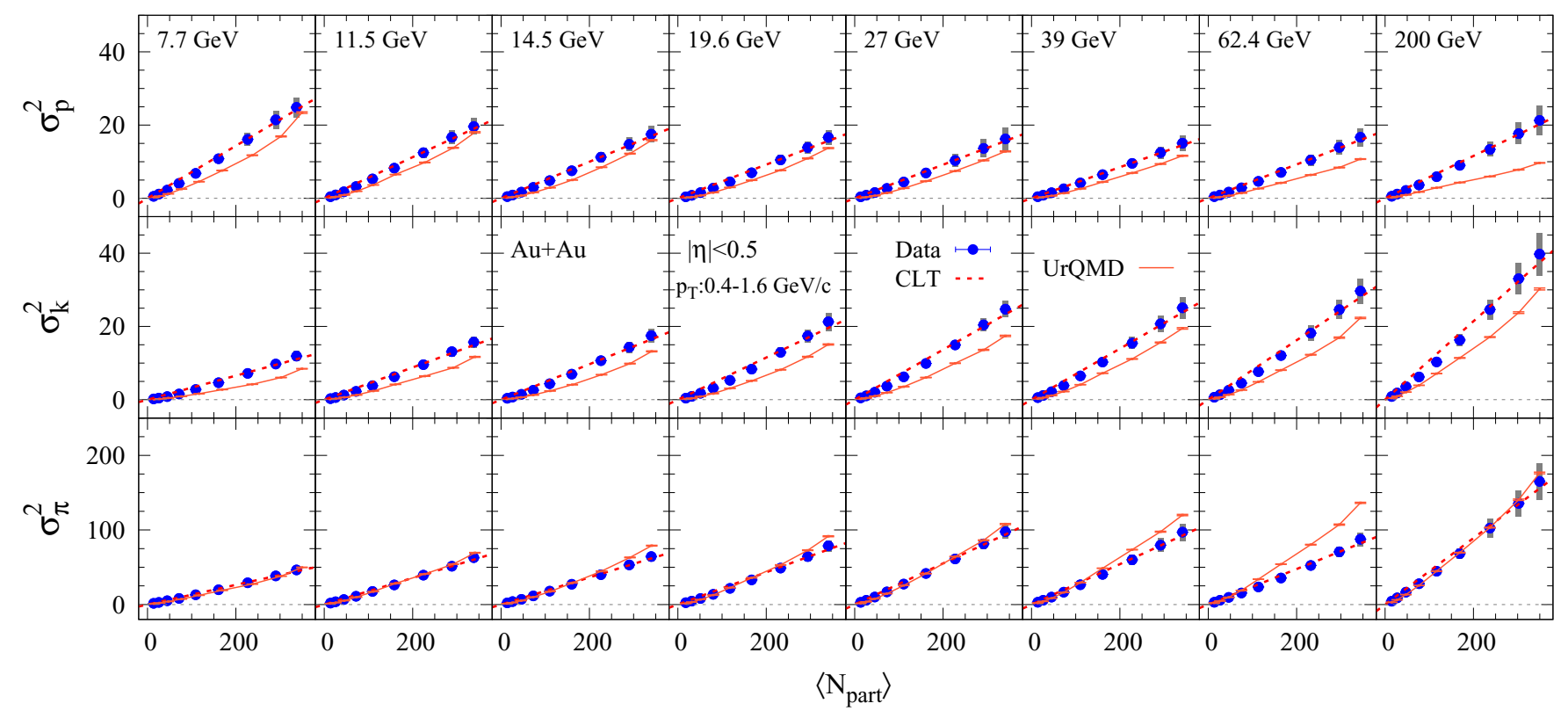

FIG. 3. Centrality dependence of efficiency-corrected second-order diagonal cumulants of net-proton, net-kaon, and net-pion (top to bottom) of the multiplicity distributions for $\mathrm{Au}+\mathrm{Au}$ collisions at $\sqrt{s_{N N}}=7.7,11.5,14.5,19.6,27,39,62.4$, and $200 \mathrm{GeV}$ (left to right) within kinematic range of $|\eta|<0.5$ and $0.4<p_{\mathrm{T}}<1.6 \mathrm{GeV} / c$. The boxes represent the systematic error. The statistical error bars are within the marker size. The dashed lines represent scaling predicted by the central limit theorem and the solid lines are UrQMD calculations.

follows:

$$
\begin{aligned}
C_{Q^{\mathrm{PI}}, p} & =\frac{\sigma_{Q^{\mathrm{PID}}, p}^{1,1}}{\sigma_{p}^{2}}=\frac{\sigma_{\pi, p}^{1,1}}{\sigma_{p}^{2}}+\frac{\sigma_{k, p}^{1,1}}{\sigma_{p}^{2}}+1, \\
C_{Q^{\mathrm{PI}}, k} & =\frac{\sigma_{Q^{\mathrm{PID}, k}}^{1,1}}{\sigma_{k}^{2}}=\frac{\sigma_{\pi, k}^{1,1}}{\sigma_{k}^{2}}+\frac{\sigma_{k, p}^{1,1}}{\sigma_{k}^{2}}+1 .
\end{aligned}
$$

Here $\sigma_{Q^{\mathrm{PI}}, p}^{1,1}=\sigma_{\pi, p}^{1,1}+\sigma_{k, p}^{1,1}+\sigma_{p}^{2}$ and $\sigma_{Q^{\mathrm{PI}}, k}^{1,1}=\sigma_{\pi, k}^{1,1}+\sigma_{p, k}^{1,1}+\sigma_{k}^{2}$. The notation "PID" is used to indicate that instead of using inclusive charged particles as in our original paper, we are using a combination of identified pions, kaons, and protons.

In this erratum we present the following figures that are updated from the same in our original paper.

Figure 2 shows the updated efficiency corrected diagonal and off-diagonal cumulants of net-pion, net-kaon, and net-proton as a function of the $\eta$-window for the $0-5 \%$ and $70-80 \%$ centrality bins, and for eight collision energies. Here we replace the results of $\sigma_{Q}^{2}, \sigma_{Q, p}^{1,1}$, and $\sigma_{Q, k}^{1,1}$ with $\sigma_{\pi}^{2}, \sigma_{\pi, p}^{1,1}$, and $\sigma_{\pi, k}^{1,1}$, respectively. The results for $\sigma_{p, k}^{1,1}, \sigma_{p}^{2}$, and $\sigma_{k}^{2}$ remain unchanged. The $\sigma_{\pi, k}^{1,1}$ and $\sigma_{\pi, p}^{1,1}$ show a linearly decreasing trend with increasing pseudorapidity acceptance window ( $\eta$-window).

In Fig. 3, the $\sigma_{Q}^{2}$ is supplanted by $\sigma_{\pi}^{2}$ that shows a linear increasing trend as a function of collision centrality and agrees well with the UrQMD calculations. The results for $\sigma_{p}^{2}$ and $\sigma_{k}^{2}$ remain unchanged.

In Fig. 4, the $\sigma_{Q, k}^{1,1}$ and $\sigma_{Q, p}^{1,1}$ are replaced by $\sigma_{\pi, k}^{1,1}$ and $\sigma_{\pi, p}^{1,1}$ respectively. The values of $\sigma_{\pi, k}^{1,1}$ and $\sigma_{\pi, p}^{1,1}$ are negative at all collision energies, which indicates $\pi-p$ and $\pi-k$ are anticorrelated.

Figure 5 shows the centrality dependence of cumulant ratios. The quantities $C_{Q^{\mathrm{PID}}, p}$ and $C_{Q^{\mathrm{PID}}, k}$ are updated using Eqs. (2) and (3), respectively. The current data points agree with UrQMD.

Figure 6 shows the collision energy dependence of $C_{p, k}, C_{Q^{\mathrm{PD}}, p}$, and $C_{Q^{\mathrm{PD}}, k}$ for $0-5 \%$ and $70-80 \%$ centralities. The results are compared with UrQMD and HRG calculations. The UrQMD calculations are redone using Eqs. (2) and (3). The quantities $C_{Q^{\mathrm{PID}}, p}$ and $C_{Q^{\mathrm{PID}}, k}$ decrease with collision energy and are below the Poisson baseline. The quantity $C_{Q^{\mathrm{PI}}, k}$ agrees well with both the UrQMD and hadron resonance gas model (HRG) calculations. The quantity $C_{Q^{\mathrm{PID}}, p}$ agrees with the UrQMD calculations but deviates from the HRG results.

In summary, we address the issue of self-correlation in the previously considered efficiency correction for (1) net-charge and net-proton and (2) net-charge and net-kaon second-order off-diagonal cumulants. For these quantities, we replace unidentified charged hadrons, as used in our original paper, with the sum of pions, kaons, and protons. Unlike our previous observations, 


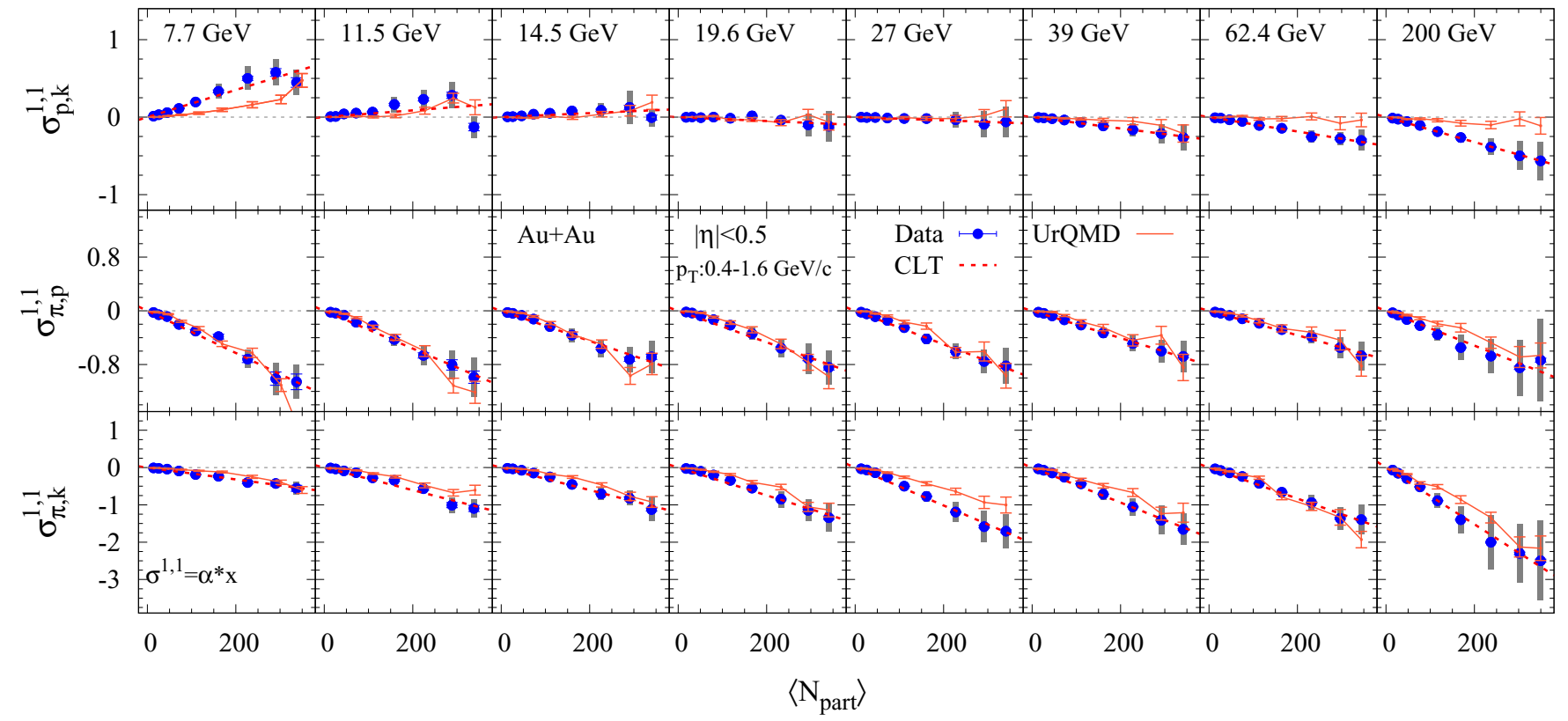

FIG. 4. Centrality dependence of second-order off-diagonal cumulants of net-proton, net-charge, and net-kaon for Au + Au collisions at $\sqrt{s_{N N}}=7.7,11.5,14.5,19.6,27,39,62.4$ and $200 \mathrm{GeV}$ (left to right) within kinematic range $|\eta|<0.5$ and $0.4<p_{\mathrm{T}}<1.6 \mathrm{GeV} / c$. Bars represent statistical errors and boxes show systematic errors. The dashed lines represent scaling predicted by the central limit theorem and the solid lines are UrQMD calculations.

we see the following differences: (1) the cumulant ratios do not show strong dependence on centrality or collision energy, (2) for the cumulant ratio of identified net-charge and net-kaon $\left(C_{Q^{\mathrm{PID}}, k}\right)$ we do not see any strong deviation from UrQMD or HRG calculations, and (3) for the identified net-charge and net-proton case $\left(C_{Q^{\mathrm{PI}}, p}\right)$ we observe that the results are slightly below the HRG calculations but are consistent with the UrQMD calculations.

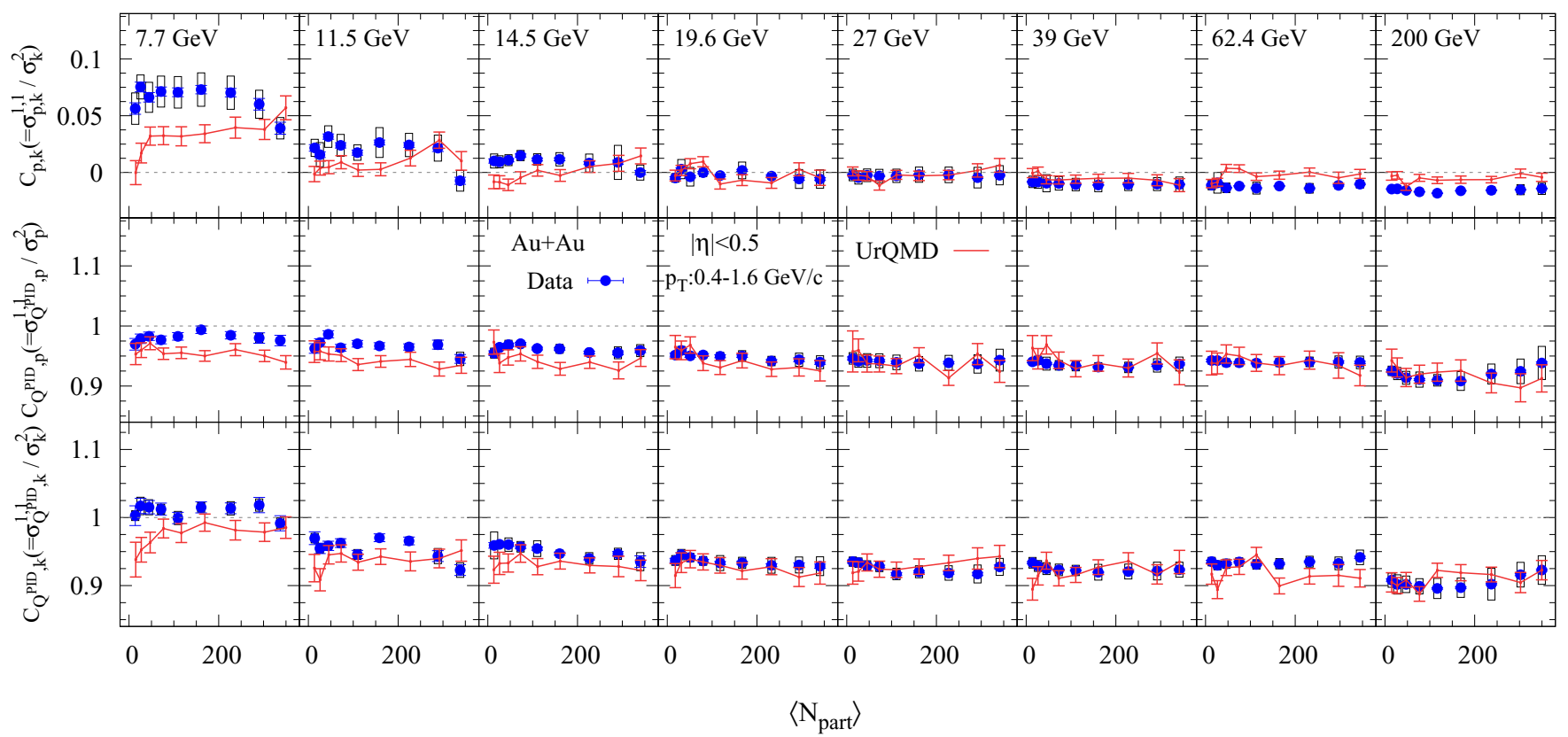

FIG. 5. Centrality dependence of second-order off-diagonal to diagonal cumulants ratios of net-proton, net-kaon, and identified net-charge for $\mathrm{Au}+\mathrm{Au}$ collisions at $\sqrt{s_{N N}}=7.7,11.5,14.5,19.6,27,39,62.4$ and $200 \mathrm{GeV}$ (left to right) within the kinematic range $|\eta|<0.5$ and $0.4<p_{\mathrm{T}}<1.6 \mathrm{GeV} / c$. Bars represent statistical errors and boxes show systematic errors. The solid lines represent the UrQMD calculations. 


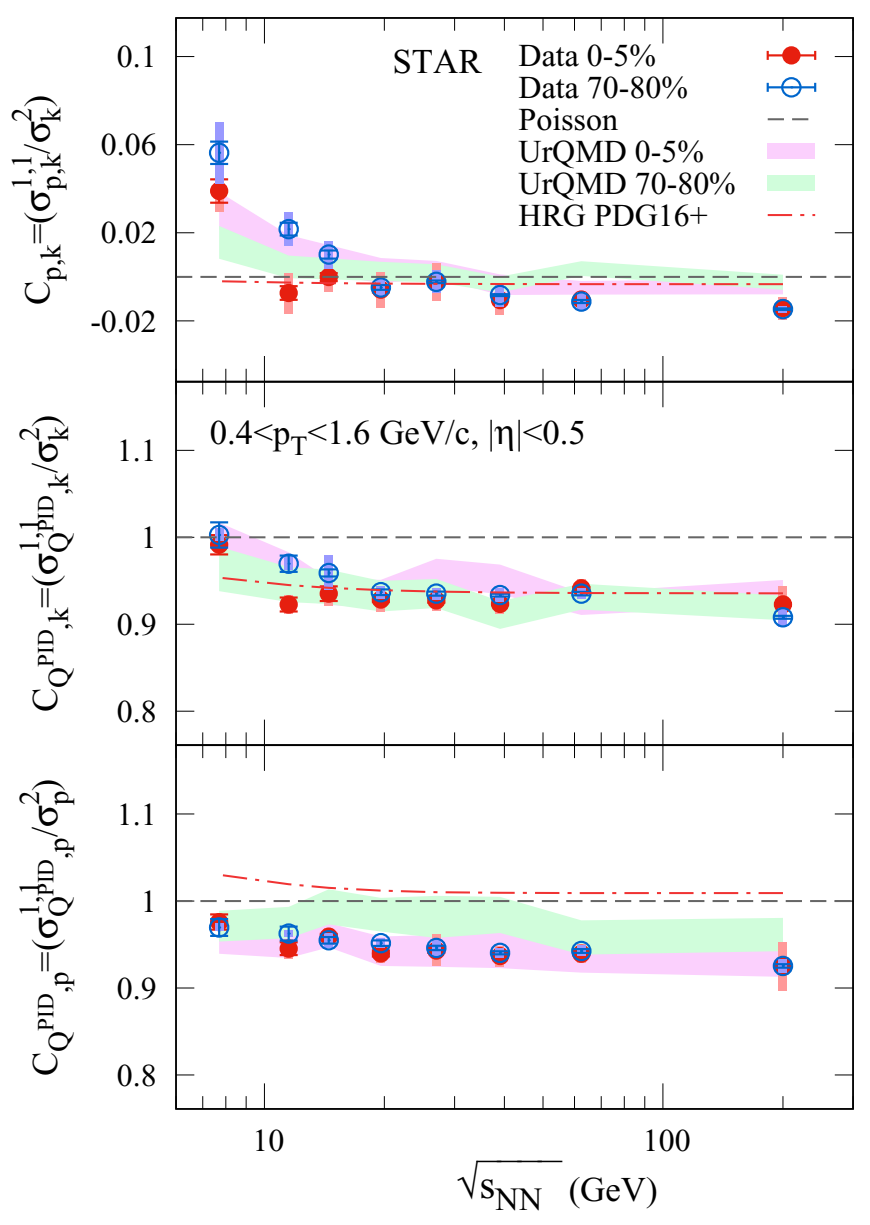

FIG. 6. Beam energy dependence of the cumulant ratios $\left(C_{p, k}, C_{Q^{\mathrm{PID}}, k}\right.$, and $C_{Q^{\mathrm{PID}}, p}$, top to bottom) of net-proton, net-kaon, and identified net-charge for $\mathrm{Au}+\mathrm{Au}$ collisions at $\sqrt{s_{N N}}=7.7,11.5,14.5,19.6,27,39,62.4$, and $200 \mathrm{GeV}$. The bands denote the UrQMD calculations for 0-5\% and 70-80\% centralities. The HRG calculations are represented by red dotted lines. The Poisson baseline is given by the black dashed lines. Bars show statistical errors and boxes show systematic errors.

We thank V. Vovchenko and V. Koch for pointing out the issues of efficiency correction with off-diagonal cumulants and important discussions. We thank the RHIC Operations Group and RCF at BNL, the NERSC Center at LBNL, and the Open Science Grid consortium for providing resources and support. This work was supported in part by the Office of Nuclear Physics within the U.S. DOE Office of Science, the U.S. National Science Foundation, the Ministry of Education and Science of the Russian Federation, National Natural Science Foundation of China, Chinese Academy of Science, the Ministry of Science and Technology of China and the Chinese Ministry of Education, the Higher Education Sprout Project by Ministry of Education at NCKU, the National Research Foundation of Korea, Czech Science Foundation and Ministry of Education, Youth and Sports of the Czech Republic, Hungarian National Research, Development and Innovation Office, New National Excellency Programme of the Hungarian Ministry of Human Capacities, Department of Atomic Energy and Department of Science and Technology of the Government of India, the National Science Centre of Poland, the Ministry of Science, Education and Sports of the Republic of Croatia, RosAtom of Russia and German Bundesministerium fur Bildung, Wissenschaft, Forschung and Technologie (BMBF), Helmholtz Association, Ministry of Education, Culture, Sports, Science, and Technology (MEXT), and Japan Society for the Promotion of Science (JSPS).

[1] V. Vovchenko and V. Koch, Nucl. Phys. A 1010, 122179 (2021).

[2] A. Bzdak and V. Koch, Phys. Rev. C 86, 044904 (2012).

[3] A. Bzdak and V. Koch, Phys. Rev. C 91, 027901 (2015).

[4] A. Chatterjee, T. Nonaka, S. Esumi, and X. Luo, Chin. Phys. C 45, 094001 (2021). 